\title{
EQUIDISTRIBUTION OF NEUMANN DATA MASS ON SIMPLICES AND A SIMPLE INVERSE PROBLEM
}

\author{
HANS CHRISTIANSON
}

\begin{abstract}
In this paper we study the behaviour of the Neumann data of Dirichlet eigenfunctions on simplices. We prove that the $L^{2}$ norm of the (semi-classical) Neumann data on each face is equal to $2 / n$ times the $(n-1)$-dimensional volume of the face divided by the volume of the simplex. This is a generalization of [Chr17] to higher dimensions. Again it is not an asymptotic, but an exact formula. The proof is by simple integrations by parts and linear algebra.

We also consider the following inverse problem: do the norms of the Neumann data on a simplex determine a constant coefficient elliptic operator? The answer is yes in dimension 2 and no in higher dimensions.
\end{abstract}

\section{INTRODUCTION}

In this paper we extend the results of [Chr17] on triangles to simplices, which are the higher dimensional analogues of triangles. The proof has many similarities but involves more linear algebra and elementary geometry. We have chosen to separate the two proofs in order to make the paper about triangles simple and clean. We also have added to this paper some applications to rudimentary inverse problems.

Let $T \subset \mathbb{R}^{n}$ be an $n$ dimensional (non-degenerate) simplex with faces $G_{0}, \ldots G_{n}$. We consider the Dirichlet eigenfunction problem on $T$ :

$$
\left\{\begin{array}{l}
-h^{2} \Delta u=u \text { in } T, \\
\left.u\right|_{\partial T}=0 .
\end{array}\right.
$$

The semiclassical parameter $h>0$ denotes the (inverse of) the eigenvalues hence takes values in a discrete set. We assume that the eigenfunctions are normalized: $\|u\|_{L^{2}(T)}=1$. Our main result, similarly to in [Chr17] is that the Neumann data on each face of the simplex is proportional to the volume of the face.

Theorem 1. Let $T \subset \mathbb{R}^{n}$ be a non-degenerate simplex with faces $G_{0}, G_{1}, \ldots, G_{n}$ and suppose u solves (1.1).

Then the (semi-classical) Neumann data on each of the the boundary faces satisfies

$$
\int_{G_{j}}\left|h \partial_{\omega} u\right|^{2} d S_{j}=\frac{2 \operatorname{Vol}_{n-1}\left(G_{j}\right)}{n \operatorname{Vol}_{n}(T)} .
$$

Here $h \partial_{\omega}$ is the semi-classical normal derivative on $\partial T, d S_{j}$ is the surface measure on $G_{j}, \operatorname{Vol}_{n}(T)$ is the volume of the simplex $T$, and $\operatorname{Vol}_{n-1}\left(G_{j}\right)$ is the $n-1$ dimensional induced volume of $G_{j}$. 
Remark 1.1. As in [Chr17, we are calling this "equidistribution" of Neumann mass since it says that the Neumann data has mass proportional to the $(n-1)$-dimensional volume of the face to which it is restricted.

The proportionality constant in (1.2) depends in a seemingly non-obvious way on the dimension $n$. However, it turns out this is the right dimensional constant in the case of the Cauchy data for quantum ergodic eigenfunctions restricted to a hypersurface, and indeed also for the boundary data quantum ergodic restriction theorems in the original studies GL93, HZ04]. One of the original motivations for the present paper was to see if one could isolate the mass of the Dirichlet vs. Neumann data of quantum ergodic eigenfunctions restricted to an interior simplex hypersurface in the Cauchy data restriction theorem in CTZ13. Unfortunately this does not help, and the present paper and Chr17 do not preclude the possibility of quantum ergodic eigenfunctions having $o(1)$ (in $L^{2}$ ) restrictions to the boundary of an interior simplex. See below for a brief history.

A statement such as Theorem 1 is false in general for other polygonal domains. It is clearly false in the case of a square, as discussed in [Chr17], as well as for a rectangular parallelepiped in any dimension by looking at Fourier series.

1.1. Brief History. Previous results on restrictions to hypersurfaces primarily focused on upper bounds. Burq-Gérard-Tzvetkov [BGT07] give an upper bound of the norm (squared) of the restrictions of eigenfunctions, of order $\mathcal{O}\left(h^{-1 / 2}\right)$. In the author's paper with Hassell-Toth [CHT13], an upper bound of $\mathcal{O}(1)$ was proved for (semi-classical) Neumann data restricted to arbitrary co-dimension 1 hypersurfaces in any dimension. Both of these estimates are shown to be sharp, so this gives a lower (and upper) bound for some eigenfunctions.

In the case of quantum ergodic eigenfunctions, a little more is known. GérardLeichtnam GL93 and Hassell-Zelditch [HZ04 give asymptotic formulae for (a density one subsequence of) the Neumann (respectively Dirichlet) boundary data of Dirichlet (respectively Neumann) quantum ergodic eigenfunctions. That means that there is a lower bound, and explicit local asymptotic formula in this special case, at least for most of the eigenfunctions. Similar statements were proved for interior hypersurfaces in [TZ12,TZ13, CTZ13. However, for an interior hypersurface, it seems an intractible problem to separate the behaviour of the Dirichlet or Neumann data, or a sparse subsequence must be removed. This again gives lower bounds on the norms of the Dirichlet or Neumann data for some of the eigenfunctions.

Acknowledgements. The work in this paper is supported in part by NSF grant DMS-1500812.

\section{The STANDARD SIMPLEX IN $\mathbb{R}^{3}$}

In this section we prove the theorem for the standard simplex in dimension 3 as it is simple to see how the proof works in this case. In Section 3 we prove the general result. 
Let $p_{0}=(0,0,0), p_{1}=(1,0,0), p_{2}=(0,1,0)$, and $p_{3}=(0,0,1)$. The standard simplex is given by all convex combinations of these vectors:

$$
T=\left\{\sum_{j=0}^{3} t_{j} p_{j}: \sum_{j=0}^{3} t_{j}=1, \text { and } t_{j} \geqslant 0\right\} .
$$

That is, $T$ is the four sided solid with the $p_{j}$ and 0 at the corners.

We use $\left(x_{1}, x_{2}, x_{3}\right)$ as the standard rectangular coordinates in $\mathbb{R}^{3}$. Let us denote $F_{1}$ denote the face in the $\left(x_{2}, x_{3}\right)$ plane (where $\left.x_{1}=0\right), F_{2}$ the face where $x_{2}=0$, $F_{3}$ the face where $x_{3}=0$, and $F_{4}$ the remaining face. Then the unit normals are $\nu_{j}=-e_{j}, j=1,2,3$ and $\nu_{4}=(3)^{-1 / 2}(1,1,1)$ respectively, where $e_{j}$ are the standard basis vectors pointing in the direction of $x_{j}$ respectively. Then the statement of the theorem involves the quantities $\left|\nu_{j} \cdot h \partial u\right|$ restricted to their respective faces.

Let us denote by $X$ the vector field

$$
X=\left(x_{1}+m_{1}\right) \partial_{x_{1}}+\left(x_{2}+m_{2}\right) \partial_{x_{2}}+\left(x_{3}+m_{3}\right) \partial_{x_{3}},
$$

where the $m_{j}$ s are parameters independent of $x$. A simple computation yields that $\left[-h^{2} \Delta-1, X\right]=-2 h^{2} \Delta$. Then the eigenfunction equation (1.1) and an application of Green's formula gives

or

$$
\begin{aligned}
\int_{T}( & {\left.\left[-h^{2} \Delta-1, X\right] u\right) \bar{u} d V } \\
& =\int_{T}\left(\left(-h^{2} \Delta-1\right) X u\right) \bar{u} d V \\
& =\int_{\partial T}\left(-h \partial_{\nu} h X u\right) \bar{u} d S+\int_{\partial T}(h X u)\left(h \partial_{\nu} \bar{u}\right) d S,
\end{aligned}
$$

$$
\begin{aligned}
2 & =2 \int_{T}|u|^{2} d V \\
& =-2 \int_{T}\left(h^{2} \Delta u\right) \bar{u} d V \\
& =\int_{\partial T}\left(-h \partial_{\nu} h X u\right) \bar{u} d S+\int_{\partial T}(h X u)\left(h \partial_{\nu} \bar{u}\right) d S \\
& =\int_{\partial T}(h X u)\left(h \partial_{\nu} \bar{u}\right) d S,
\end{aligned}
$$

since we have assumed Dirichlet boundary conditions.

Let us break the analysis into the four different faces. On $F_{1}$, we have

$$
\begin{aligned}
\int_{F_{1}} & (h X u)\left(h \partial_{\nu} \bar{u}\right) d S \\
\quad= & \int_{F_{1}}\left(\left(\left(x_{1}+m_{1}\right) h \partial_{x_{1}}+\left(x_{2}+m_{2}\right) h \partial_{x_{2}}+\left(x_{3}+m_{3}\right) h \partial_{x_{3}}\right) u\right) \bar{u} d S_{1} \\
\quad= & -m_{1} \int_{F_{1}}\left|h \partial_{\nu_{1}} u\right|^{2} d S_{1},
\end{aligned}
$$


since $h \partial_{x_{1}}=-h \partial_{\nu_{1}}$ and $h \partial_{x_{j}}$ is tangential when $j=2,3$. Similarly, for $j=2,3$ we have

$$
\int_{F_{j}}(h X u)\left(h \partial_{\nu_{j}} \bar{u}\right) d S_{j}=-m_{j} \int_{F_{j}}\left|h \partial_{\nu_{j}}\right|^{2} d S_{j}
$$

On $F_{4}$ we need to be a little bit more careful. The points on $F_{4}$ all satisfy $x_{1}+$ $x_{2}+x_{3}=1$ since the normal is parallel to $(1,1,1)$. The normal derivative is $h \partial_{\nu_{4}}=$ $3^{-1 / 2}\left(h \partial_{x_{1}}+h \partial_{x_{2}}+h \partial_{x_{3}}\right)$, and the tangent vectors are all linear combinations of $e_{3}-e_{1}=(-1,0,0)$ and $e_{2}-e_{1}=(-1,1,0)$, so that

$$
\partial_{x_{j}}=3^{-1 / 2} \partial_{\nu_{4}}
$$

for $j=1,2,3$. Hence

$$
\begin{aligned}
\int_{F_{4}} & (h X u) h \partial_{\nu_{4}} \bar{u} d S_{4} \\
& =\int_{F_{4}}\left(\left(\left(x_{1}+m_{1}\right) h \partial_{x_{1}}+\left(x_{2}+m_{2}\right) h \partial_{x_{2}}+\left(x_{3}+m_{3}\right) h \partial_{x_{3}}\right) u\right) h \partial_{\nu_{4}} \bar{u} d S_{4} \\
& =(3)^{-1 / 2} \int_{F_{4}}\left(\left(\left(x_{1}+m_{1}\right)+\left(x_{2}+m_{2}\right)+\left(x_{3}+m_{3}\right)\right) h \partial_{\nu_{4}} u\right) h \partial_{\nu_{4}} \bar{u} d S_{4} \\
& =3^{-1 / 2}\left(1+m_{1}+m_{2}+m_{3}\right) \int_{F_{4}}\left|h \partial_{\nu_{4}} u\right|^{2} d S_{4} .
\end{aligned}
$$

Summing up, we have

$$
\begin{aligned}
2 & =-m_{1} \int_{F_{1}}\left|h \partial_{\nu_{1}} u\right|^{2} d S_{1}-m_{2} \int_{F_{2}}\left|h \partial_{\nu_{2}} u\right|^{2} d S_{2}-m_{3} \int_{F_{3}}\left|h \partial_{\nu_{3}} u\right|^{2} d S_{3} \\
& +3^{-1 / 2}\left(1+m_{1}+m_{2}+m_{3}\right) \int_{F_{4}}\left|h \partial_{\nu_{4}} u\right|^{2} d S_{4} .
\end{aligned}
$$

Now if $m_{j}=0$ for $j=1,2,3$, using (2.3) we have

$$
2=(3)^{-1 / 2} \int_{F_{4}}\left|h \partial_{\nu_{4}} u\right|^{2} d S_{4}
$$

so that

$$
\int_{F_{4}}\left|h \partial_{\nu_{4}} u\right|^{2} d S_{4}=3^{1 / 2} \cdot 2
$$

We know that $\operatorname{Vol}_{3}(T)=1 / 3 !=1 / 6$. The cross product computes the area of the parallelogram, which is twice the area of the triangle, so that tells us that

$$
\begin{aligned}
\operatorname{Vol}_{2}\left(F_{4}\right) & =|(-1,1,0) \times(-1,0,1)| / 2 \\
& =\sqrt{3} / 2 .
\end{aligned}
$$


Hence

$$
\begin{aligned}
\int_{F_{4}}\left|h \partial_{\nu_{4}} u\right|^{2} d S_{4} & =2 \cdot 3^{1 / 2} \\
& =4(\sqrt{3} / 2) \\
& =(2 / 3)\left(\frac{2 \cdot 3^{1 / 2}}{1 / 6}\right) \\
& =\frac{2 \operatorname{Vol}_{2}\left(F_{4}\right)}{n \operatorname{Vol}_{3}(T)}
\end{aligned}
$$

For $j=1,2,3$ we have

$$
\mathrm{Vol}_{2}\left(F_{j}\right)=1 / 2
$$

Differentiating (2.3) with respect to $m_{j}$, we have

or

$$
0=-\int_{F_{j}}\left|h \partial_{\nu_{j}} u\right|^{2} d S_{j}+(3)^{-1 / 2} \int_{F_{4}}\left|h \partial_{\nu_{4}} u\right|^{2} d S_{4},
$$

$$
\begin{aligned}
2 & =\int_{F_{j}}\left|h \partial_{\nu_{j}} u\right|^{2} d S_{j} \\
& =\left(\frac{2}{3}\right)\left(\frac{1 / 2}{1 / 6}\right) \\
& =\left(\frac{2}{3}\right) \frac{\operatorname{Vol}_{2}\left(F_{j}\right)}{\operatorname{Vol}_{3}(T)} .
\end{aligned}
$$

This proves the theorem for the standard simplex in dimension 3 .

\section{Proof of Theorem 1}

Let $p_{1}, \ldots, p_{n}$ be independent vectors in $\mathbb{R}^{n}$, and let $p_{0}=(0, \ldots, 0)$ denote the origin. Then

$$
T=\left\{\sum_{0}^{n} t_{j} p_{j}: \sum t_{j}=1 \text { and } t_{j} \geqslant 0\right\}
$$

is a simplex. If $p_{j}=e_{j}$ (standard rectangular basis vectors) for each $j$, then we say $T$ is the standard simplex and denote it by $T_{0}$.

Since the $p_{j}$ s are independent, the matrix

$$
A=\left[\begin{array}{cccc}
\mid & \mid & \cdots & \mid \\
p_{1} & p_{2} & \cdots & p_{n} \\
\mid & \mid & \cdots & \mid
\end{array}\right]
$$

is invertible. Let $B=A^{-1}$, and for $x \in \mathbb{R}^{n}$ set

$$
y=B x .
$$

This transformation simply takes the simplex $T$ to the standard simplex $T_{0}$. Indeed, if $x=p_{j}$, then $B x=e_{j}$. Hence

$$
T_{0}=\left\{\sum t_{j} B p_{j}, \sum t_{j}=1, t_{j} \geqslant 0 \forall j\right\} .
$$


We pause briefly to point out that this change of variables induces a volume element, so that

$$
\operatorname{det}(A)=n ! \operatorname{Vol}(T)
$$

This is easily seen using the volume of the standard simplex is $1 / n$ ! and the Jacobian for a change of volume integral is $\operatorname{det}(A)$.

We lift the transformation to $T^{*} \mathbb{R}^{n}$ : for $\xi \in \mathbb{R}^{n}$, let $\eta=\left(B^{-1}\right)^{T} \xi$. Then since the symbol of the Laplacian in $\mathbb{R}^{n}$ is $\xi_{1}^{2}+\ldots+\xi_{n}^{2}$, the symbol for the Laplacian in our new coordinates is

$$
\xi^{T} \xi=\eta^{T} B B^{T} \eta
$$

Set $\Gamma=B B^{T}$ and

$$
-h^{2} \widetilde{\Delta}=-\sum \Gamma_{i j} \partial_{y_{i}} \partial_{y_{j}}
$$

the Laplacian in the $y$ coordinates on the standard simplex $T_{0}$.

For the eigenfunctions $u$ on $T$, let $v(y)=u(A y)$ be the eigenfunctions in the $y$ coordinates. Since $-h^{2} \widetilde{\Delta}$ is constant coefficient, the same commutator argument can be used here. Indeed, let

$$
Y=\sum\left(y_{j}+m_{j}\right) \partial_{y_{j}}
$$

and a simple calculation gives

$$
\left[-h^{2} \widetilde{\Delta}-1, Y\right]=-2 h^{2} \widetilde{\Delta}
$$

Following the recipe in Section 2, we have using $-h^{2} \widetilde{\Delta} v=v$ and Green's formula

$$
\begin{aligned}
2 \int_{T_{0}}|v|^{2} d y= & -2 \int_{T_{0}}\left(h^{2} \widetilde{\Delta} v\right) \bar{v} d y \\
= & \int_{T_{0}}\left(\left[-h^{2} \widetilde{\Delta}-1, Y\right] v\right) \bar{v} d y \\
= & \int_{T_{0}}\left(\left(-h^{2} \widetilde{\Delta}-1\right) Y v\right) \bar{v} d y \\
= & \int_{T_{0}}\left(\left(-(h \partial)^{T} B B^{T} h \partial-1\right) Y v\right) \bar{v} d y \\
= & \int_{T_{0}}\left(B B^{T} h \partial Y v\right) \cdot(h \partial \bar{v}) d y-\int_{T_{0}}(Y v) \bar{v} d y \\
& +\int_{\partial T_{0}}\left(-\nu^{T} B B^{T} h \partial(h Y v)\right) \bar{v} d y \\
= & \int_{T_{0}}\left(B B^{T} h \partial Y v\right) \cdot(h \partial \bar{v}) d y-\int_{T_{0}}(Y v) \bar{v} d y
\end{aligned}
$$


since we have assumed Dirichlet boundary condtions. Here $\nu$ denotes the unit outward normal and $d S$ denotes the induced surface measure. Continuing,

$$
\begin{aligned}
2 \int_{T_{0}}|v|^{2} d y= & \int_{T_{0}}\left(B B^{T} h \partial Y v\right) \cdot(h \partial \bar{v}) d y-\int_{T_{0}}(Y v) \bar{v} d y \\
= & \int_{T_{0}}(Y v)\left(-h \partial^{T} B B^{T} h \partial \bar{v}\right) d y-\int_{T_{0}}(Y v) \bar{v} d y \\
& +\int_{\partial T_{0}}(h Y v)\left(\nu^{T} B B^{T} h \partial \bar{v}\right) d S \\
= & \int_{\partial T_{0}}(h Y v)\left(\nu^{T} B B^{T} h \partial \bar{v}\right) d S
\end{aligned}
$$

since $\widetilde{\Delta} \bar{v}=\bar{v}$.

We have changed variables to be on $T_{0}$ in order to make sure the normal vectors are easy to compute. For $T_{0}$, let $F_{j}$ be the side where $y_{j}=0,1 \leqslant j \leqslant n$, and $F_{0}$ the remaining face. Then for $1 \leqslant j \leqslant n$, we have the outgoing normal vectors to $F_{j}$ $\nu_{j}=-e_{j}$, where the $e_{j}$ are the standard basis vectors. For $F_{0}$, we have transformed to $T_{0}$ so that

$$
\nu_{0}=n^{-1 / 2}(1, \ldots, 1)
$$

Then the unit normal derivatives are

$$
h \partial_{\nu_{j}}=-h \partial_{y_{j}}
$$

for $1 \leqslant j \leqslant n$ and

$$
h \partial_{\nu_{0}}=n^{-1 / 2}\left(h \partial_{x_{1}}+\ldots+h \partial_{x_{n}}\right) .
$$

We are assuming Dirichlet boundary conditions, so all of the tangential derivatives of $v$ vanish. That is, for $1 \leqslant j \leqslant n$,

$$
h \partial_{\ell} v=0,
$$

except for $\ell=j$. We also have using symmetry that on $F_{0}$,

$$
h \partial_{\nu_{0}} v=n^{1 / 2} h \partial_{y_{j}} v
$$

for every $1 \leqslant j \leqslant n$. We recall again that $y_{j}=0$ on $F_{j}$ for $1 \leqslant j \leqslant n$ and on $F_{0}$ we have $y_{1}+y_{2}+\ldots+y_{n}=1$. 
Plugging these observations in to (3.2), we have

$$
\begin{aligned}
2 \int_{T_{0}}|v|^{2} d y= & \int_{\partial T_{0}}(h Y v)\left(\nu^{T} B B^{T} h \partial \bar{v}\right) d S \\
= & \sum_{j=1}^{n} \int_{F_{j}}\left(\left(\sum_{\ell}\left(m_{\ell}+y_{\ell}\right) h \partial_{y_{\ell}}\right) v\right)\left(\nu_{j}^{T} B B^{T} h \partial \bar{v}\right) d S_{j} \\
& +\int_{F_{0}}\left(\left(\sum_{\ell}\left(m_{\ell}+y_{\ell}\right) h \partial_{y_{\ell}}\right) v\right)\left(\nu_{0}^{T} B B^{T} h \partial \bar{v}\right) d S_{0} \\
= & \sum_{j=1}^{n} \int_{F_{j}}\left(m_{j} h \partial_{y_{j}} v\right)\left(h \partial_{\nu_{j}} \bar{v}\right) d S_{j} \\
& +\int_{F_{0}}\left(\sum_{1}^{n}\left(n^{-1 / 2}\left(y_{j}+m_{j}\right)\right) h \partial_{\nu_{0}} v\right)\left(\nu_{0}^{T} B B^{T} h \partial \bar{v}\right) d S_{0} \\
= & \sum_{j=1}^{n} \int_{F_{j}}\left(-m_{j} h \partial_{\nu_{j}} v\right)\left(\nu_{j}^{T} B B^{T} h \partial \bar{v}\right) d S_{j} \\
& +\int_{F_{0}} n^{-1 / 2}\left(1+m_{1}+\ldots+m_{n}\right)\left(\left(h \partial_{\nu_{0}}\right) v\right)\left(\nu_{0}^{T} B B^{T} h \partial \bar{v}\right) d S_{0} \\
= & \sum_{j=1}^{n}\left(-m_{j}\right) I_{j}+n^{-1 / 2}\left(1+m_{1}+\ldots+m_{n}\right) I_{0}
\end{aligned}
$$

where for each $0 \leqslant j \leqslant n$

$$
I_{j}=\int_{F_{j}}\left(h \partial_{\nu_{j}} v\right)\left(\nu_{j}^{T} B B^{T} h \partial \bar{v}\right) d S_{j} .
$$

Let us now compute the $I_{j}$ s. Using equation (3.3), setting $m_{j}=0$ for all $1 \leqslant j \leqslant n$, we have

$$
I_{0}=2 n^{1 / 2} \int_{T_{0}}|v|^{2} d y
$$

Differentiating equation (3.3) with respect to $m_{j}$ yields for $1 \leqslant j \leqslant n$

$$
I_{j}=n^{-1 / 2} I_{0}=2 \int_{T_{0}}|v|^{2} d y .
$$

Now we must compute the $I_{j}$ in terms of the corresponding integrals on the original simplex $T$. We first observe that, since for $1 \leqslant j \leqslant n$ we have $F_{j} \subset\left\{y_{j}=0\right\}$, changing variables on one of the boundary integrals induces the area of the $(n-1)$-dimensional parallelepiped spanned by $p_{1}, p_{2}, \ldots, p_{j-1}, p_{j+1}, \ldots, p_{n}$. Denote this parallelepiped $\Gamma_{j}$, and observe that

$$
\operatorname{Vol}_{n-1} \Gamma_{j}=(n-1) ! \operatorname{Vol}_{n-1}\left(G_{j}\right),
$$

where $G_{j}$ is the $(n-1)$-dimensional simplex spanned by $p_{1}, p_{2}, \ldots, p_{j-1}, p_{j+1}, \ldots, p_{n}$.

For $F_{0}$, our area element is $n^{1 / 2} d y$, so changing variables in the integral over $F_{0}$ induces the area of the parallelepiped spanned by $p_{1}, p_{2}-p_{1}, p_{3}-p_{1}, \ldots, p_{n}-p_{1}$ 
divided by $n^{1 / 2}$. Denote this parallelepiped by $\Gamma_{0}$, and again we have

$$
\operatorname{Vol}_{n-1}\left(\Gamma_{0}\right)=(n-1) ! \operatorname{Vol}_{n-1}\left(G_{0}\right) .
$$

We now need to compute the integrand inside of each $I_{j}$ in terms of the corresponding normal derivatives on $G_{j}$ of $u$.

We first observe that on $F_{j}$, for $1 \leqslant j \leqslant n, h \partial_{y_{\ell}} \bar{v}=0$ for $\ell \neq j$, so that the semiclassical gradient can be written

$$
\left.h \partial_{y} v\right|_{F_{j}}=\left.e_{j} h \partial_{y_{j}} v\right|_{F_{j}}=\left.\nu_{j} h \partial_{\nu_{j}} v\right|_{F_{j}} .
$$

Similarly, for $j=0$, we have on $F_{0}$

$$
\begin{aligned}
h \partial v & =\left[\begin{array}{c}
h \partial_{y_{1}} \\
\vdots \\
h \partial_{y_{n}}
\end{array}\right] v \\
& =n^{-1 / 2}\left[\begin{array}{c}
1 \\
\vdots \\
1
\end{array}\right] h \partial_{\nu_{0}} v \\
& =\nu_{0} h \partial_{\nu_{0}} v
\end{aligned}
$$

Now for each $j$, let $\omega_{j}$ be the unit outward normal on $G_{j}$. We know for each $j$ on the face $G_{j}$

$$
\begin{aligned}
\left.h \partial_{\omega_{j}} u\right|_{G_{j}} & =\left.\omega_{j}^{T} h \partial_{x} u\right|_{G_{j}} \\
& =\left.\omega_{j}^{T} B^{T} h \partial_{y} v\right|_{F_{j}} \\
& =\left.\left(B \omega_{j}\right)^{T} h \partial_{y} v\right|_{F_{j}} \\
& =\left.\left(B \omega_{j}\right)^{T} \nu_{j} h \partial_{\nu_{j}} v\right|_{F_{j}} \\
& =\left.\left(\omega_{j}^{T} B^{T} \nu_{j}\right) h \partial_{\nu_{j}} v\right|_{F_{j}},
\end{aligned}
$$

so that

$$
\left.h \partial_{\nu_{j}} v\right|_{F_{j}}=\left.\left(\omega_{j}^{T} B^{T} \nu_{j}\right)^{-1} h \partial_{\omega_{j}} u\right|_{G_{j}}
$$

written in the $y$ and $x$ coordinates respectively.

On the other hand, we have $h \partial_{x}=B^{T} h \partial_{y}$, so that

$$
\nu_{j}^{T} B h \partial_{x} u=\nu_{j}^{T} B B^{T} h \partial_{y} v .
$$

The left hand side is zero except for the projection on to the $\omega_{j}$, so that on each $G_{j}$ we have

$$
\begin{aligned}
\nu_{j}^{T} B h \partial_{x} u & =\left(\nu_{j}^{T} B \omega_{j}\right) \omega_{j}^{T} h \partial_{x} u \\
& =\left(\omega_{j}^{T} B^{T} \nu_{j}\right) h \partial_{\omega_{j}} u .
\end{aligned}
$$

Hence

$$
\left.\nu_{j}^{T} B B^{T} h \partial_{y} v\right|_{F_{j}}=\left.\left(\omega_{j}^{T} B^{T} \nu_{j}\right) h \partial_{\omega_{j}} u\right|_{G_{j}}
$$


Plugging these observations in to the formulae for the $I_{j}$, we get for $1 \leqslant j \leqslant n$

$$
\begin{aligned}
I_{j} & =\int_{F_{j}}\left(h \partial_{\nu_{j}} v\right)\left(\nu_{j}^{T} B B^{T} h \partial \bar{v}\right) d S_{j} \\
& =\frac{1}{(n-1) ! \operatorname{Vol}_{n-1}\left(G_{j}\right)} \int_{G_{j}}\left(\left.\left(\omega_{j}^{T} B^{T} \nu_{j}\right)^{-1} h \partial_{\omega_{j}} u\right|_{G_{j}}\right)\left(\left.\left(\omega_{j}^{T} B^{T} \nu_{j}\right) h \partial_{\omega_{j}} \bar{u}\right|_{G_{j}}\right) d \widetilde{S}_{j} \\
& =\frac{1}{(n-1) ! \operatorname{Vol}_{n-1}\left(G_{j}\right)} \int_{G_{j}}\left|h \partial_{\omega_{j}} u\right|^{2} d \widetilde{S}_{j},
\end{aligned}
$$

where $d \widetilde{S}_{j}$ is the induced surface measure on $G_{j}$.

On the other hand, for $I_{0}$, we have

$$
\begin{aligned}
I_{0} & =\int_{F_{0}}\left(h \partial_{\nu_{0}} v\right)\left(\nu_{0}^{T} B B^{T} h \partial \bar{v}\right) d S_{0} \\
& =\frac{n^{1 / 2}}{(n-1) ! \operatorname{Vol}_{n-1}\left(G_{0}\right)} \int_{G_{0}}\left|\partial_{\omega_{0}} u\right|^{2} d \widetilde{S}_{0},
\end{aligned}
$$

where $d \widetilde{S}_{0}$ is the induced surface measure on $G_{0}$.

We recall that

$$
\int_{T_{0}}|v|^{2} d y=\frac{1}{n ! \operatorname{Vol}_{n}(T)}
$$

so that rearranging we have for each $1 \leqslant j \leqslant n$

$$
I_{j}=\frac{2}{n ! \operatorname{Vol}_{n}(T)}
$$

and

$$
I_{0}=n^{1 / 2} \frac{2}{n ! \operatorname{Vol}_{n}(T)} .
$$

Rearranging, we have for $0 \leqslant j \leqslant n$

$$
\begin{array}{rl}
\int_{G_{j}} & \left.h \partial_{\omega_{j}} u\right|^{2} d \widetilde{S}_{j} \\
& =\frac{2(n-1) ! \operatorname{Vol}_{n-1}\left(G_{j}\right)}{n ! \operatorname{Vol}_{n}(T)} \\
& =\frac{2 \operatorname{Vol}_{n-1}\left(G_{j}\right)}{n \operatorname{Vol}_{n}(T)}
\end{array}
$$

which completes the proof of Theorem 1 ,

\section{A Simple inVERSE PRoblem}

The proof of Theorem 1 suggests a further question: If $u$ solves a constant coefficient eigenfunction equation, does the Neumann data determine the coefficients? In fact, in this paper, we only have information about the norms of the Neumann data, so we cannot fully answer this question using only this very elementary information. In fact, in the general case, the answer is that the norms of the Neumann data do not determine the coefficients (see Subsection 4.1 below). However, in dimension 2 the 
norms do determine the coefficients. We will return to this question after a few easier results.

This question is, of course intimately related to posing the standard Laplacian eigenfunction problem on a different simplex. Let us pose it as such in dimension 2. Let $T \subset \mathbb{R}^{2}$ be a triangle with sides $a, b, c$, with the convention that the length of the sides are $a, b, c$ respectively. Suppose $u$ solves

$$
\left\{\begin{array}{l}
\left(-h^{2} \Delta-1\right) u=0 \text { on } T, \\
\left.u\right|_{\partial T}=0, \\
\|u\|_{L^{2}(T)} .
\end{array}\right.
$$

We have the following Theorem.

Theorem 2. Suppose u solves (4.1), and suppose $N_{a}=\int_{a}\left|h \partial_{\nu} u\right|^{2} d S$ and similarly for $N_{b}$ and $N_{c}$. Then the three quantities $N_{a}, N_{b}, N_{c}$ uniquely determine the triangle $T$ (up to reflection).

This theorem seems obvious, but in the formulae for the $N_{a}, N_{b}$, and $N_{c}$, there is both the length of the side and the area of the triangle. The proof is by scaling.

Proof. Suppose we have another triangle $T_{1}$ with the same Neumann data norms. Let $a_{1}, b_{1}, c_{1}$ denote the three sides of $T_{1}$, again with the convention that $a_{1}, b_{1}, c_{1}$ denote also the length of the sides. We know that the Neumann data relates the lengths of the sides to the area of the triangle. We have

$$
N_{a}=\frac{a}{\operatorname{Area}(T)},
$$

and similarly for $b, c$. On the other hand, we also have

$$
N_{a}=\frac{a_{1}}{\operatorname{Area}\left(T_{1}\right)},
$$

and similarly for $b_{1}, c_{1}$. Equating these quantities, we have

$$
\frac{a}{a_{1}}=\frac{\operatorname{Area}(T)}{\operatorname{Area}\left(T_{1}\right)},
$$

and similarly

$$
\frac{b}{b_{1}}=\frac{c}{c_{1}}=\frac{\operatorname{Area}(T)}{\operatorname{Area}\left(T_{1}\right)} .
$$

This means that the side lengths of $T_{1}$ are all scalar multiples of the corresponding sides on $T$ with the same scalar. Hence $T_{1}$ is similar to $T$. Let

$$
\lambda=\frac{\operatorname{Area}(T)}{\operatorname{Area}\left(T_{1}\right)} .
$$

On the one hand, this implies that

$$
\operatorname{Area}(T)=\lambda \operatorname{Area}\left(T_{1}\right)
$$

On the other hand, we have

$$
a=\lambda a_{1}
$$

and similarly

$$
b=\lambda b_{1}, \quad c=\lambda c_{1} .
$$


As the lengths scale linearly, the area scales quadratically. That is, (4.3) and (4.4) imply that

$$
\operatorname{Area}(T)=\lambda^{2} \operatorname{Area}\left(T_{1}\right)
$$

Hence combining with (4.2), we have $\lambda^{2}=\lambda$, so that $\lambda=1$. This means precisely that $T=T_{1}$ (up to reflection).

We now consider the question of determining the coefficients of a constant coefficient elliptic operator on the standard 2-simplex. Let $B$ be a non-degenerate $2 \times 2$ matrix, and let $\Gamma=B B^{T}$. Consider $P=-\Gamma_{i j} h \partial_{x_{i}} h \partial_{x_{j}}$ be the associated positive definite elliptic operator. Our next result is that the semi-classical Neumann data uniquely determines the operator $P$. Interestingly, this does not determine the matrix $B$ (see Remark 4.4).

Theorem 3. Let $B$ be a non-degenerate $2 \times 2$ matrix and $\Gamma=B B^{T}$. Let $P=$ $-\Gamma_{i j} h \partial_{x_{i}} h \partial_{x_{j}}$. Let $T_{0}$ be the standard triangle in $\mathbb{R}^{2}$ generated by the vectors $(1,0)$ and $(0,1)$. Suppose u solves the eigenfunction problem

$$
\left\{\begin{array}{l}
P u=u \text { in } T_{0}, \\
\left.u\right|_{\partial T_{0}}=0, \\
\|u\|_{L^{2}\left(T_{0}\right)}=1 .
\end{array}\right.
$$

Let $F_{1}$ and $F_{2}$ denote the sides of length 1 and $F_{0}$ the hypotenuse of length $\sqrt{2}$. Then the norms

$$
\left\|h \partial_{\nu} u\right\|_{L^{2}\left(F_{1}\right)}^{2},\left\|h \partial_{\nu} u\right\|_{L^{2}\left(F_{2}\right)}^{2}, \text { and }\left\|h \partial_{\nu} u\right\|_{L^{2}\left(F_{0}\right)}^{2}
$$

uniquely determine $\Gamma$.

Remark 4.1. We pause to remark that in the statement of the theorem is buried a rather astounding fact: the norms of the (semi-classical) Neumann data of any single eigenfunction determine $\Gamma$. Of course this requires some knowledge also about the spectrum. In other words, if one eigenvalue and corresponding eigenfunction's Neumann mass is known, then $\Gamma$ is uniquely determined.

Remark 4.2. It is also very interesting that the proof in fact computes the entries of $\Gamma$ explicitly in terms of the Neumann data norms. Indeed, if we label

$$
J_{1}=\left\|h \partial_{\nu} u\right\|_{L^{2}\left(F_{1}\right)}^{2}, J_{2}=\left\|h \partial_{\nu} u\right\|_{L^{2}\left(F_{2}\right)}^{2},
$$

and

$$
J_{0}=\left\|h \partial_{\nu} u\right\|_{L^{2}\left(F_{0}\right)}^{2},
$$

and we write $\Gamma=(\Gamma)_{j k}$, we have

$$
\begin{aligned}
& \Gamma_{11}=\frac{2}{J_{1}}, \\
& \Gamma_{22}=\frac{2}{J_{2}},
\end{aligned}
$$

and

$$
\Gamma_{12}=\Gamma_{21}=\frac{2 \sqrt{2}}{J_{0}}-\frac{1}{J_{1}}-\frac{1}{J_{2}} .
$$


In particular, if $J_{1}=J_{2}=2$ and $J_{0}=2 \sqrt{2}$, we have $\Gamma=I$ as expected (since each $J_{j}$ is twice the length of the sides, which is the length of the side divided by the area of the triangle).

First we write a Lemma giving yet another way of computing the Neumann data mass. We state this Lemma in any dimension.

Lemma 4.3. Let $B$ be a non-degenerate $n \times n$ matrix, $\Gamma=B B^{T}$, and

$$
P=-\Gamma_{i j} h \partial_{x_{i}} h \partial_{x_{j}} .
$$

Let $T_{0}$ be the standard simplex in $\mathbb{R}^{n}$ with faces $F_{0}, F_{1}, \ldots, F_{n}$ in the notation of earlier in this paper. Suppose u solves the eigenfunction problem

$$
\left\{\begin{array}{l}
P u=u \text { in } T_{0} \\
\left.u\right|_{\partial T_{0}}=0 \\
\|u\|_{L^{2}\left(T_{0}\right)}=1
\end{array}\right.
$$

Then on each face $F_{j}, 0 \leqslant j \leqslant n$, we have

$$
\int_{F_{j}}\left(h \partial_{\nu_{j}} u\right)\left(\nu_{j}^{T} B B^{T} h \partial_{x} \bar{u}\right) d S_{j}=\left|B^{T} \nu_{j}\right|^{2} \int_{F_{j}}\left|h \partial_{\nu_{j}} u\right|^{2} d S_{j},
$$

where $d S_{j}$ is the induced surface measure on $F_{j}$ as usual.

Note that this is a different way of computing this quantity than in (3.4).

Proof. We observe that on $F_{j}$, for $1 \leqslant j \leqslant n, h \partial_{x_{\ell}} \bar{u}=0$ for $\ell \neq j$, so that

$$
h \partial_{x} u=e_{j} h \partial_{x_{j}} u=\nu_{j} h \partial_{\nu_{j}} u \text {. }
$$

Similarly, for $j=0$, we have on $F_{0}$

$$
\begin{aligned}
h \partial u & =\left[\begin{array}{c}
h \partial_{y_{1}} \\
\vdots \\
h \partial_{y_{n}}
\end{array}\right] u \\
& =n^{-1 / 2}\left[\begin{array}{c}
1 \\
\vdots \\
1
\end{array}\right] h \partial_{\nu_{0}} u \\
& =\nu_{0} h \partial_{\nu_{0}} u .
\end{aligned}
$$

Then on each face $F_{j}$ with normal $\nu_{j}$, we have

$$
\begin{aligned}
\nu_{j}^{T} B B^{T} h \partial \bar{u} & =\nu_{j}^{T} B B^{T} \nu_{j} h \partial_{\nu_{j}} \bar{u} \\
& =\left(B^{T} \nu_{j}\right)^{T}\left(B^{T} \nu_{j}\right) h \partial_{\nu_{j}} \bar{u} \\
& =\left|B^{T} \nu_{j}\right|^{2} h \partial_{\nu_{j}} \bar{u} .
\end{aligned}
$$

Hence on each face $F_{j}$, we have

$$
\int_{F_{j}}\left(h \partial_{\nu_{j}} u\right)\left(\nu_{j}^{T} B B^{T} h \partial \bar{u}\right) d S_{j}=\left|B^{T} \nu_{j}\right|^{2} \int_{F_{j}}\left|h \partial_{\nu_{j}} u\right|^{2} d S_{j} .
$$

This completes the proof. 
Proof of Theorem 3. The proof proceeds by using an eigenvector diagonalization argument. It is interesting that, although the argument uses the existence of eigenvalues/vectors of $\Gamma$, we do not need to know them.

Let $v_{1}, v_{2}$ be orthonormal eigenvectors for $\Gamma$. Since $\Gamma=B B^{T}$ is positive definite, write $\lambda_{1}^{2}, \lambda_{2}^{2}$ for the eigenvalues of $\Gamma$ so that $\Gamma v_{j}=\lambda_{j}^{2} v_{j}$ for $j=1,2$. Let

$$
L=\left(\begin{array}{cc}
\mid & \mid \\
v_{1} & v_{2} \\
\mid & \mid
\end{array}\right) \text {, }
$$

so that (since $L$ is orthogonal),

$$
L^{T} \Gamma L=\left(\begin{array}{cc}
\lambda_{1}^{2} & 0 \\
0 & \lambda_{2}^{2}
\end{array}\right)
$$

Let us denote

$$
G=L\left(\begin{array}{cc}
\lambda_{1} & 0 \\
0 & \lambda_{2}
\end{array}\right)
$$

so that $G G^{T}=\Gamma$.

We now change variables using the matrix $G$. Let $T_{1}$ denote the triangle spanned by the new coordinates $v_{1}, v_{2}$. Rescaling in each variable $v_{j} \mapsto \lambda_{j}^{-1} v_{j}$ gives a new triangle $T$. Let $w(x)=u(G x)$, so that

$$
\int_{T}|w|^{2} d V=\int_{T}|u(G x)|^{2} d V=|G|^{-1} \int_{T_{0}}|u|^{2} d V=\frac{1}{\lambda_{1} \lambda_{2}} .
$$

We also have $-h^{2} \Delta w=w$ on $T_{0}$, so we can use the same commutator argument as above to compute the mass of the Neumann data. For $j=1,2$, let $I_{j}=\int_{\lambda_{j}^{-1} v_{j}}\left|h \partial_{\nu} w\right|^{2} d S$, and $I_{0}=\int_{H}\left|h \partial_{\nu} w\right|^{2} d S$ be the Neumann mass of the function $w$ on the legs spanned by the $\lambda_{j}^{-1} v_{j}$ and the hypotenuse $H$. Using Theorem 1 and (4.6), we have for $j=1,2$

$$
\begin{aligned}
I_{j} & =\left(\frac{1}{\lambda_{1} \lambda_{2}}\right)\left(\frac{\text { length of } \lambda_{j}^{-1} v_{j}}{\operatorname{area}(T)}\right) \\
& =\left(\frac{1}{\lambda_{1} \lambda_{2}}\right)\left(\frac{\lambda_{j}^{-1}}{\left(\lambda_{1}^{-1} \lambda_{2}^{-1} / 2\right)}\right) \\
& =\frac{2}{\lambda_{j}} .
\end{aligned}
$$

Further,

$$
I_{0}=2\left(\lambda_{1}^{-2}+\lambda_{2}^{-2}\right)^{1 / 2} .
$$

For $j=0,1,2$, let

$$
J_{j}=\int_{F_{j}}\left|h \partial_{\nu} u\right|^{2} d S
$$

be the Neumann mass of the original eigenfunction $u$ on the faces of $T_{0}$. These are the quantities we are assuming we know. 
Using Lemma 4.3, that means

$$
\begin{aligned}
J_{j} & =\frac{1}{\left|G^{T} \nu\right|^{2}} \int_{F_{j}}\left(h \partial_{\nu} u\right)\left(\nu^{T} G G^{T} h \nabla \bar{u}\right) d S \\
& =\left(\frac{1}{\left|G^{T} \nu\right|^{2}}\right)\left(\frac{\text { length of } F_{j}}{\text { length of } \lambda_{j}^{-1} v_{j}}\right) I_{j} \\
& =\left(\frac{1}{\left|G^{T} \nu\right|^{2}}\right) \lambda_{j}\left(\frac{2}{\lambda_{j}}\right) \\
& =\frac{2}{\left|G^{T} \nu\right|^{2}}
\end{aligned}
$$

for $j=1,2$, and

$$
J_{0}=\frac{2 \sqrt{2}}{\left|G^{T} \nu\right|^{2}} .
$$

We pause momentarily to recall that the normal vectors $\nu$ in the above expressions are the normals to the original faces $F_{j}, j=0,1,2$ on the standard triangle $T_{0}$.

Recall that $\nu_{1}=(-1,0), \nu_{2}=(0,-1)$ and $\nu_{0}=(\sqrt{2})^{-1}(1,1)$, which will help us determine the matrix $\Gamma$.

Write

$$
\Gamma=\left(\begin{array}{ll}
\Gamma_{11} & \Gamma_{12} \\
\Gamma_{21} & \Gamma_{22}
\end{array}\right)
$$

As $\Gamma$ is symmetric, we have $\Gamma_{12}=\Gamma_{21}$, so we only need to determine the three numbers $\Gamma_{11}, \Gamma_{12}$, and $\Gamma_{22}$.

The quantities we need to examine are all of the form $\left|G^{T} \nu_{j}\right|^{2}$, which we rewrite:

$$
\begin{aligned}
\left|G^{T} \nu_{j}\right|^{2} & =\left(G^{T} \nu_{j}\right)^{T}\left(G^{T} \nu_{j}\right) \\
& =\nu_{j}^{T} G G^{T} \nu_{j} \\
& =\nu_{j}^{T} \Gamma \nu_{j} .
\end{aligned}
$$

Plugging in the $\nu_{j}, j=0,1,2$, we have:

$$
\begin{aligned}
\nu_{1}^{T} \Gamma \nu_{1} & =(-1,0) \Gamma\left(\begin{array}{c}
-1 \\
0
\end{array}\right) \\
& =\Gamma_{11},
\end{aligned}
$$

and similarly

$$
\nu_{2}^{T} \Gamma \nu_{2}=\Gamma_{22} .
$$


For $\nu_{0}$, we get information about the off diagonal terms as well:

$$
\begin{aligned}
\nu_{0}^{T} \Gamma \nu_{0} & =\frac{1}{2}(1,1) \Gamma\left(\begin{array}{c}
1 \\
1
\end{array}\right) \\
& =\frac{1}{2}(1,1)\left(\begin{array}{c}
\Gamma_{11}+\Gamma_{12} \\
\Gamma_{21}+\Gamma_{22}
\end{array}\right) \\
& =\frac{1}{2}\left(\Gamma_{11}+\Gamma_{12}+\Gamma_{21}+\Gamma_{22}\right) \\
& =\frac{1}{2}\left(\Gamma_{11}+2 \Gamma_{12}+\Gamma_{22}\right)
\end{aligned}
$$

again due to $\Gamma$ being symmetric.

Returning now to (4.7), we have for $j=1,2$

$$
\begin{aligned}
J_{j} & =\frac{2}{\left|G^{T} \nu_{j}\right|^{2}} \\
& =\frac{2}{\Gamma_{j j}} .
\end{aligned}
$$

Hence

$$
\Gamma_{11}=\frac{2}{J_{1}}
$$

and similarly for $\Gamma_{22}$. For $\Gamma_{12}$, we appeal to equation (4.8) to get

$$
\begin{aligned}
J_{0} & =\frac{2 \sqrt{2}}{\left|G^{T} \nu_{0}\right|^{2}} \\
& =\frac{2 \sqrt{2}}{\frac{1}{2}\left(\Gamma_{11}+2 \Gamma_{12}+\Gamma_{22}\right)} \\
& =\frac{4 \sqrt{2}}{\Gamma_{11}+2 \Gamma_{12}+\Gamma_{22}} .
\end{aligned}
$$

Rearranging, we have

$$
\Gamma_{11}+2 \Gamma_{12}+\Gamma_{22}=\frac{4 \sqrt{2}}{J_{0}}
$$

so that solving for $\Gamma_{12}$, we have

$$
\Gamma_{12}=\frac{2 \sqrt{2}}{J_{0}}-\frac{1}{2}\left(\Gamma_{11}+\Gamma_{22}\right)
$$

Plugging in the known values of $\Gamma_{11}$ and $\Gamma_{22}$, we have

$$
\Gamma_{12}=\frac{2 \sqrt{2}}{J_{0}}-\frac{1}{2}\left(\frac{2}{J_{1}}+\frac{2}{J_{2}}\right)=\frac{2 \sqrt{2}}{J_{0}}-\frac{1}{J_{1}}-\frac{1}{J_{2}} .
$$

This gives the $\Gamma_{j k}$ in terms of the known quantities $J_{1}, J_{2}$, and $J_{0}$, completing the proof.

Remark 4.4. It is interesting to note that the proof of Theorem 3 does not uniquely determine the matrix $B$, due to rotational invariance. Indeed, if

$$
B=\left(\begin{array}{ll}
a & b \\
c & d
\end{array}\right)
$$


with $a=c=d=2^{-1 / 2}$ and $b=-2^{-1 / 2}$, then we still have $a^{2}+c^{2}=b^{2}+d^{2}=1$, and $a c+b d=0$. Note, however, that $B B^{T}=I$ in this case as well.

4.1. Dimension 3: an example. The result in Theorem 3 is false in higher dimensions, even for small perturbations of $I$. Let $T_{0}$ be the standard simplex in $\mathbb{R}^{3}, B$ be a $3 \times 3$ non-degenerate matrix, $\Gamma=B B^{T}$, and $P=-\Gamma_{i j} h \partial_{x_{i}} h \partial_{x_{j}}$. Suppose $u$ solves the eigenfunction problem (4.5). Lemma 4.3 still applies, with $\nu_{j}=-e_{j}$ for $1 \leqslant j \leqslant 3$ and $\nu_{0}=3^{-1 / 2}(1,1,1)$. For $0<\epsilon<1$, define the matrix $B$ by

$$
B^{T}=\left(\begin{array}{ccc}
a & 0 & 0 \\
d & \left(1-\epsilon^{2}\right)^{1 / 2} & \epsilon \\
\epsilon & \epsilon & \left(1-\epsilon^{2}\right)^{1 / 2}
\end{array}\right),
$$

where

$$
d=\frac{-3 \epsilon\left(1-\epsilon^{2}\right)^{1 / 2}-\epsilon^{2}}{\left(1-\epsilon^{2}\right)^{1 / 2}+\epsilon}
$$

and

$$
a=\left(1-d^{2}-\epsilon^{2}\right)^{1 / 2} .
$$

Observe that $B=I+\mathcal{O}(\epsilon)$ and satisfies

$$
\begin{aligned}
& \left|B^{T} e_{1}\right|^{2}=a^{2}+d^{2}+\epsilon^{2}=1, \\
& \left|B^{T} e_{2}\right|^{2}=\left(1-\epsilon^{2}\right)+\epsilon^{2}=1, \\
& \left|B^{T} e_{3}\right|^{2}=\epsilon^{2}+\left(1-\epsilon^{2}\right)=1,
\end{aligned}
$$

and

$$
\begin{aligned}
\left|B^{T}(1,1,1)^{T}\right|^{2}= & a^{2}+\left(d+\left(1-\epsilon^{2}\right)^{1 / 2}+\epsilon\right)^{2}+\left(2 \epsilon+\left(1-\epsilon^{2}\right)^{1 / 2}\right)^{2} \\
= & a^{2}+d^{2}+\left(1-\epsilon^{2}\right)+\epsilon^{2}+2 d\left(1-\epsilon^{2}\right)^{1 / 2}+2 d \epsilon+2 \epsilon\left(1-\epsilon^{2}\right)^{1 / 2} \\
& +4 \epsilon^{2}+\left(1-\epsilon^{2}\right)+4 \epsilon\left(1-\epsilon^{2}\right)^{1 / 2} \\
= & \left(1-\epsilon^{2}\right)+\left(1-\epsilon^{2}\right)+\epsilon^{2}+2 d\left(\left(1-\epsilon^{2}\right)^{1 / 2}+\epsilon\right) \\
& +2 \epsilon\left(1-\epsilon^{2}\right)^{1 / 2}+4 \epsilon^{2}+\left(1-\epsilon^{2}\right)+4 \epsilon\left(1-\epsilon^{2}\right)^{1 / 2} \\
= & 2-\epsilon^{2}-6 \epsilon\left(1-\epsilon^{2}\right)^{1 / 2}-2 \epsilon^{2}+6 \epsilon\left(1-\epsilon^{2}\right)^{1 / 2}+1+3 \epsilon^{2} \\
= & 3 .
\end{aligned}
$$

These are the same values one gets from $B=I=\Gamma$, however $B B^{T} \neq I$, so these 4 numbers do not determine $\Gamma$.

\section{REFERENCES}

[BGT07] N. Burq, P. Gérard, and N. Tzvetkov. Restrictions of the Laplace-Beltrami eigenfunctions to submanifolds. Duke Math. J., 138(3):445-486, 2007.

[Chr17] Hans Christianson. Equidistribution of neumann data mass on triangles. preprint, 2017.

[CHT13] Hans Christianson, Andrew Hassell, and John A. Toth. Semiclassical control and $L^{2}$ restriction bounds for neumann data along hypersurfaces. Int. Math. Res. Not. IMRN, to appear, 2013.

[CTZ13] Hans Christianson, John A. Toth, and Steve Zelditch. Quantum ergodic restriction for Cauchy data: interior que and restricted que. Math. Res. Lett., 20(3):465-475, 2013. 
[GL93] Patrick Gérard and Éric Leichtnam. Ergodic properties of eigenfunctions for the Dirichlet problem. Duke Math. J., 71(2):559-607, 1993.

[HZ04] Andrew Hassell and Steve Zelditch. Quantum ergodicity of boundary values of eigenfunctions. Comm. Math. Phys., 248(1):119-168, 2004.

[TZ12] J.A. Toth and S. Zelditch. Quantum ergodic restriction theorems, i: interior hypersurfaces in domains with ergodic billiards. Annales Henri Poincaré, 13:599-670, 2012.

[TZ13] John A. Toth and Steve Zelditch. Quantum ergodic restriction theorems: manifolds without boundary. Geom. Funct. Anal., 23(2):715-775, 2013.

(H. Christianson) Department of Mathematics, University of North Carolina.

E-mail address: hans@math.unc.edu 